\title{
Contrast-enhanced ultrasound is better than magnetic resonance imaging in evaluating the short-term results of microwave ablation treatment of uterine fibroids
}

\author{
YAN ZHANG, MEIWU ZHANG, XIAOXIANG FAN and DAFENG MAO \\ Department of Interventional Therapy, Ningbo No. 2 Hospital, Ningbo, Zhejiang 315010, P.R. China
}

Received September 1, 2016; Accepted August 1, 2017

DOI: $10.3892 / \mathrm{etm} .2017 .5171$

\begin{abstract}
Contrast-enhanced ultrasound (CEUS) and magnetic resonance imaging (MRI) were compared in evaluating the short-term effects of microwave ablation (MWA) on uterine fibroids. A total of 60 patients with uterine fibroids treated by MWA were enrolled in the experimental group during their two-year follow-up period according to the inclusion criteria. Conventional two-dimensional US, MRI and CEUS were performed to determine the volume reduction and the fibroid residue by displaying the size, echo and signal intensity of fibroids prior to and after MWA treatment. As the control group, 60 consecutive patients were recruited on their follow-up visit at least two years after MWA treatment of uterine fibroids. Significant differences were observed in the wash-in rate (WiR) of the fibroid tissue, start time difference, rise time ratio (RTR) and WiR ratio between the experimental and control groups $(\mathrm{P}<0.05)$. However, the WiR of fibroid vessel, total area under the curve of fibroid vessel and tissue, and rise time difference (RTD) between fibroid vessel and tissue did not display any significant differences between the two groups. Fibroids were either reduced in volume or cured by MWA therapy in patients with uterine fibroids. The reductions in volume of hypointense, isointense and hyperintense fibroids were $62.42 \pm 18.13,53.27 \pm 10.05$ and $47.43 \pm 9.56 \%$, respectively, on T1-weighted imaging (T1WI). On T2WI, the corresponding reductions were $67.32 \pm 32.63,59.36 \pm 19.36$ and $42.63 \pm 10.37 \%$, respectively. The higher the signal intensity on T1WI and $\mathrm{T} 2 \mathrm{WI}$, the lower the reduction in volume. It is indicative that different blood supply to fibroids results in different ablation. CEUS was proved to be more effective than MRI in evaluating the effects of MWA on uterine fibroids during the first postoperative year.
\end{abstract}

Correspondence to: Professor Yan Zhang, Department of Interventional Therapy, Ningbo No. 2 Hospital, 42 Yongfeng West Road, Haishu, Ningbo, Zhejiang 315010, P.R. China

E-mail: zyan333@126.com

Key words: contrast-enhanced ultrasound, magnetic resonance imaging, uterine fibroids, microwave ablation

\section{Introduction}

Uterine fibroids are the most common benign tumor type of the female genital tract in women of reproductive age (1). It mostly occurs in women between of 35 and 50 years of age and the estimated prevalence in the United States is $20-40 \%$ (2). The most frequent clinical symptoms are menorrhagia, lower abdominal pain, a sensation of pelvic fullness and infertility $(3,4)$. Available treatments are medication, surgery (including hysterectomy and myomectomy) and less invasive therapies. Surgeries may cause severe trauma and undesirable co-morbidities (5). Medical therapy only an effect on the clinical symptoms based on long-term administration, and has adverse effects (6). A large variety of less invasive alternatives to hysterectomy have been developed, such as uterine artery embolization (UAE), high-intensity focused ultrasound (HIFU) and radiofrequency thermal ablation (7-9). Exposure to X-ray radiation is a persistent problem of UAE and may cause numerous complications (7). HIFU may be time-consuming and less effective in large uterine fibroids despite its non-invasiveness (8). Microwave ablation (MWA) has a high thermal efficiency and has gained wide use in treating solid tumors in organs other than the uterus $(10,11)$.

MWA has now become an important alternative for treating uterine fibroids, and is usually performed under the guidance of ultrasound (US) and magnetic resonance imaging (MRI). MWA has several advantages over other available techniques; for instance, it is non-invasive, feasible and safe, and it only requires a short downtime with no radiation-induced injuries (12). It is of great value in treating patients with gynecological diseases who require non-invasive treatments with organ function preservation $(12,13)$. However, optimal imaging techniques are important in evaluating the necrosis of fibroids after MWA.

US is one of the most commonly used techniques for detecting uterine fibroids. However, conventional US has limitations in assessing the mid- and long-term results of treatments (14). Contrast-enhanced US (CEUS) has become the focus of medical research on US and has become more reliable in evaluating the effects of MWA due to the improvement of high-frequency probe and color Doppler flow imaging (CDFI) techniques (14). Furthermore, MRI is considered to be the most precise imaging technique to reach a diagnosis of uterine fibroids as well as to locate and evaluate the lesion; it is 
also able to distinguish uterine fibroids from adenomyosis (15). Furthermore, the signal intensity on T2-weighted imaging (T2WI) provides abundant information on uterine fibroids.

In the present study, CEUS was performed on enrolled patients with uterine fibroids to assess the effectiveness of MWA in treating uterine fibroids.

\section{Patients and methods}

Patients. Between October 2013 and September 2015, a total of 60 consecutive patients with uterine fibroids who received MWA treatment at Ningbo No. 2 Hospital (Ningbo, China), who were in their two-year follow-up period, were enrolled in the experimental group. The inclusion criteria were as follows: i) Female patients of reproductive age, diagnosed with uterine fibroids by US and MRI, who had completed childbearing, declined hysterectomy and experienced symptoms such as menorrhagia, abdominal pain, pelvic pain and anemia; ii) pre-menopausal women with no desire for fertility; iii) intramural or subserous myoma of $>5 \mathrm{~cm}$ in diameter or submucous myoma with a diameter of $>3 \mathrm{~cm}$, or an uterine junctional zone of $>5 \mathrm{~mm}$ in width and a lesion of $>3 \mathrm{~cm}$ in thickness; iv) patients with complete data from conventional and enhanced MRI examinations pre- and post-MWA therapy; and v) no history of treatments including UEA and HIFU.

As the control group, 60 consecutive patients were recruited on their follow-up visit at least two years after MWA treatment for uterine fibroids. The common clinical symptoms were progressive dysmenorrhea, prolonged menstruation, menorrhagia and pelvic bulge. Of the 78 fibroids included in the control group, 57 were single and 21 were multiple (Table I). The diameter of the fibroid ranged from 1.5 to $4.0 \mathrm{~cm}$ with a mean of $3.2 \mathrm{~cm}$.

The Institutional Review Boards of Ningbo No. 2 Hospital approved the present study and all participants provided written informed consent prior to enrollment.

CEUS examination. Ultrasonography was performed using an Acuson Sequoia 512 US system (Siemens Medical Solutions, Mountain View, CA, USA) with the mechanical index varying from 0.15 to 0.20 . The frequency of the transducers ranged from 2.0 to $4.0 \mathrm{MHz}$. The contrast agent used for CEUS was SonoVue (Bracco Imaging Co., Gorizia, Italy) dissolved in $5 \mathrm{ml} 0.9 \%$ sodium chloride solution. Gray-scale US was first performed to identify the morphology, location, size, echo and resistance index of uterine fibroids. Subsequently, CEUS was performed for a continuous observation $>3$ min after a rapid intravenous bolus administration of contrast medium through the antecubital vein. The fibroid volume was calculated using the following formula: $V=1 / 6 \pi x D 1 x D 2 x D 3(16)$, where D1, D2 and D3 are the longitudinal, transverse and anteroposterior diameters of the fibroid, respectively, on CEUS. Two experienced sonographers reviewed the images.

Sonographic evaluation was repeated at one week prior to MWA therapy and at one and six months as well as one and two years following the procedure.

MRI examination. Images were acquired on a 3.0T system (GE Signa Profile; GE Healthcare, Little Chalfont, UK). The imaging protocols included a T1W spin echo (SE) sequence
Table I. Demographic and clinical data of patients with uterine fibroids prior to treatment.

\begin{tabular}{lcc}
\hline Parameter & $\begin{array}{c}\text { Experimental } \\
\text { group }(\mathrm{n}=60)\end{array}$ & $\begin{array}{c}\text { Control } \\
\text { group }(\mathrm{n}=60)\end{array}$ \\
\hline Age, years (range) & $\begin{array}{c}45.8 \pm 6.7 \\
(38-55)\end{array}$ & $\begin{array}{c}40.3 \pm 15.7 \\
(30-60)\end{array}$ \\
$\begin{array}{l}\text { Clinical symptoms (\%) } \\
\text { Progressive }\end{array}$ & $60(100)$ & $59(98.3)$ \\
dysmenorrhea & & \\
Menorrhagia & $56(93.3)$ & $52(56.6)$ \\
Pelvic bulge & $49(81.6)$ & $41(68.3)$ \\
Prolonged menstruation & $42(70.0)$ & $37(61.6)$ \\
Shortened menstruation & $34(56.6)$ & $28(46.6)$ \\
Fibroid location $(\%)$ & & \\
Anterior wall & $53(88.3)$ & $50(83.3)$ \\
Cervix & $10(16.6)$ & $12(20.0)$ \\
Posterior wall & $15(25.0)$ & $16(26.6)$ \\
\hline
\end{tabular}

Values are expressed as the mean \pm standard deviation and range or as $\mathrm{n}(\%)$.

with a repetition time (TR) of $360 \mathrm{msec}$ and an echo time (TE) of $16 \mathrm{msec}$, and a T2W SE sequence with TR of 3,000 msec and a TE of $90 \mathrm{msec}$ in transverse and sagittal planes (slice thickness, $1 \mathrm{~mm}$; field of view, $34 \mathrm{~mm}$; number of excitations, 4; matrix, 256x192).

MRI was performed in all patients at one week prior to the procedure and at one and six months as well as one and two years following the procedure.

MWA procedure. MWA therapy was performed using a microwave device (KV2000; Kangyou Microwave Energy Sources Institute, Nanjing, China) with a frequency of 2,450 $\mathrm{MHz}$. The patient adopted a lithotomy position and underwent the procedure after local anesthesia. A single percutaneous microwave electrode was rapidly placed into the fibroids under UA guidance. The output energy of the microwave was set between 50 and $60 \mathrm{~W}$, while the treatment time was set at 480-1,440 sec, according to the volume and sonographic changes of the fibroid. In a three-dimensional manner, the electrode was placed from shallow to deep and from back to front. The ablation region involved the fibroids and the normal tissue within $1 \mathrm{~cm}$ around the lesion to prevent recurrence (17).

Perfusion parameters on CEUS and MRI. The perfusion parameters derived from the time-intensity curve (TIC) included mean transit time, start time (ST), rise time (RT), peak enhancement $(\mathrm{PE})$, wash-in rate $(\mathrm{WiR})$ and total area under the curve (AUC) during the enhancement period of fibroid tissue and vessel, the ST, RT and AUC differences between fibroid vessel and tissue, and the RT, PE, WiR and AUC ratios of fibroid vessel to tissue. CEUS was repeated at different postoperative times to assess the enhancement inside or around the lesion at different phases. Pre-operative enhanced MRI was applied to determine the enhancement 
Table II. Quantitative perfusion parameters derived from the time-intensity curve.

\begin{tabular}{lccc}
\hline Parameter & Experimental group & Control group & T-value \\
\hline Fibroid tissue & & & P-value \\
MTT (sec) & $9.32 \pm 2.62$ & $13.21 \pm 4.35$ & -3.562 \\
AUC & $64.32 \pm 25.36$ & $70.32 \pm 36.23$ & -0.465 \\
WiR & $1.43 \pm 0.36$ & $0.92 \pm 0.46$ & 2.732 \\
Fibroid vessel & & & $0.036^{\mathrm{a}}$ \\
AUC & $136.56 \pm 48.32$ & $149.36 \pm 69.62$ & $0.027^{\mathrm{a}}$ \\
WiR & $3.21 \pm 1.22$ & $3.37 \pm 1.36$ & -0.163 \\
RTR & $1.35 \pm 0.19$ & $1.96 \pm 0.24$ & 1.362 \\
WiRR & $0.56 \pm 0.23$ & $0.29 \pm 0.13$ & 2.763 \\
RTD (sec) & $1.32 \pm 0.92$ & $3.56 \pm 1.12$ & -1.362 \\
STD (sec) & $1.32 \pm 0.32$ & $2.32 \pm 0.92$ & -1.369 \\
\hline
\end{tabular}

${ }^{\text {a }}<0$ 0.05. Values are expressed as the mean \pm standard deviation. MTT, mean transit time; AUC, total area under the curve; WiR, wash-in rate; RTR, rise time ratio of vessel to tissue in fibroids; WiRR, WiR ratio of vessel to tissue in fibroids; RTD, rise time difference between vessel and tissue in fibroids; STD, start time difference between vessel and tissue in fibroids.

inside the targeted area. After the operation, MRI was repeated to detect necrosis and residues of fibroids.

Adverse events. Adverse events during the MWA therapy were recorded, and they included pain in the sacrococcygeal, hip and targeted regions as well as skin burns. Immediately after the therapy, vital signs, skin conditions, the amount and color of vaginal discharge and the activity of extremities were examined. Patients were followed up during the first postoperative month to record any adverse events.

Statistical analysis. Data were analyzed using SPSS 17.0 statistical software (SPSS, Inc., Chicago, IL, USA). Values are expressed as the mean \pm standard deviation. Student's t-test was performed to compare between groups and the chi-square test was used to analyze enumeration data. $\mathrm{P}<0.05$ was considered to indicate a statistically significant difference.

\section{Results}

Characteristics and parameters of uterine fibroids on CEUS. Prior to MWA therapy, CEUS was performed to investigate the variations of parameters during different phases. As presented in Table II, significant differences were observed in the WiR of the fibroid tissue, STD, RTR and WiR ratio between the experimental and control groups. However, the WiR of fibroid vessels, AUC of fibroid vessels and tissues and RTD did not differ significantly between the two groups. Parameters on pre-operative CEUS may provide reliable information on the perfusion of uterine fibroids to help evaluate the regression area and remnants of fibroid objectively.

Volume and signal intensity changes of fibroids on MRI after MWA therapy. All clinical symptoms were alleviated or removed, and fibroids were either reduced in volume or cured by MWA in patients with uterine fibroids. As presented in Table III, the reductions in volume of hypointense, isointense and hyperintense fibroids were $62.42 \pm 18.13,53.27 \pm 10.05$ and
Table III. Reductions in volume of hypointense, isointense and hyperintense uterine fibroids on T1WI and T2WI.

\begin{tabular}{lccc}
\hline Signal type & $\begin{array}{c}\text { Experimental } \\
\text { group }(\%)\end{array}$ & $\begin{array}{c}\text { Control } \\
\text { group (\%) }\end{array}$ & P-value \\
\hline T1WI & & & \\
Hypointense & $62.42 \pm 18.13$ & $53.12 \pm 21.27$ & $<0.01^{\mathrm{a}}$ \\
Isointense & $53.27 \pm 10.05$ & $47.13 \pm 9.12$ & 0.07 \\
Hyperintense & $47.43 \pm 9.56$ & $40.23 \pm 10.35$ & 0.09 \\
T2WI & & & \\
Hypointense & $67.32 \pm 32.63$ & $55.08 \pm 13.37$ & $0.02^{\mathrm{a}}$ \\
Isointense & $59.36 \pm 19.36$ & $54.66 \pm 17.24$ & 0.13 \\
Hyperintense & $42.63 \pm 10.37$ & $34.84 \pm 13.19$ & 0.06 \\
\hline
\end{tabular}

${ }^{\text {a }}<<0.05$. Values are expressed as the mean \pm standard deviation. T1WI, T1-weighted imaging.

$47.43 \pm 9.56 \%$, respectively, on T1WI. On T2WI, the reductions were $67.32 \pm 32.63,59.36 \pm 19.36$ and $42.63 \pm 10.37 \%$ in hypointense, isointense and hyperintense fibroids, respectively. The higher the signal intensity on T1WI and T2WI, the lower the reduction in volume. It is indicative that different blood supply for fibroids results in different ablation. The reductions were significantly higher in the experimental group than that in the control group.

Adverse events. Among 60 patients in the experimental group, $32(53.3 \%)$ and $26(43.3 \%)$ patients complained about pain at the treated site and in the sacrococcygeal region, respectively, while 7 patients experienced radiating pain during the MWA therapy. Skin burns were reported in 38 cases $(63.4 \%)$ and improved after focus position adjustment, treatment rhythm control and water cooling. After the therapy, lower abdominal and sacrococcygeal pain were reported in $9(15.0 \%)$ and 

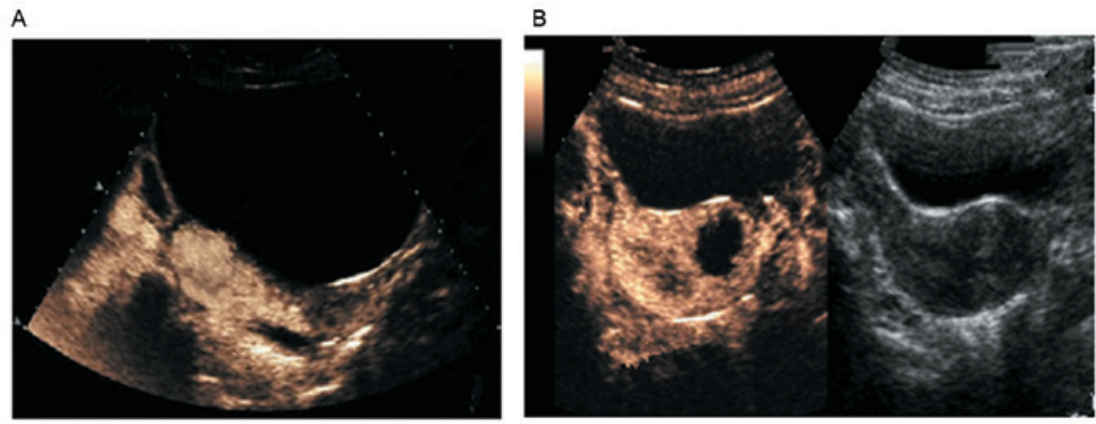

C
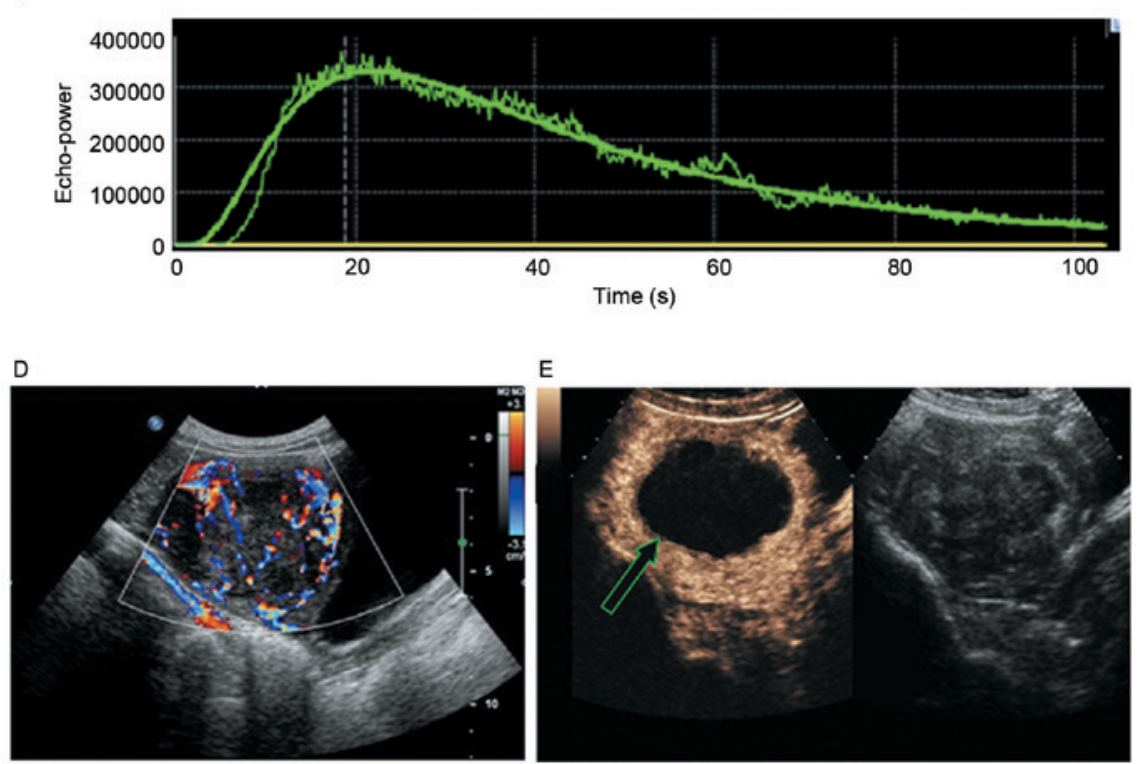

Figure 1. US images of (A-C) a patient from the experimental and (D and E) a patient from the control group. (A) CEUS performed one week prior to MWA revealed the complete contrast filling inside the fibroid. (B) Flake contrast perfusion was found at the periphery region of the fibroid on CEUS performed one month after MWA, indicating fibroid residue. (C) The time-intensity curve presented a rapid wash-in and slow wash-out enhancement pattern. (D) Color Doppler flow imaging displayed a striped and circular blood signal inside the fibroid, suggesting abundant blood supply. (E) No contrast perfusion and residue were present inside the fibroid on CEUS half a year after MWA (arrow). The fibroid was completely ablated. CEUS, contrast-enhanced ultrasound; MWA, microwave ablation.

$4(6.6 \%)$ cases, and disappeared one week later. There were no major complications, indicating that MWA is reliable for treating uterine fibroids due to its noninvasiveness.

Comparison of CEUS and MRI in pre- and post-MWA assessment. CEUS clearly revealed the non-perfused area of the fibroids prior to and after MWA (Fig. 1). In a representative subject from the experimental group, CEUS performed one week prior to MWA displayed complete contrast perfusion inside the fibroids with distinct boundaries, regular shape and homogeneous echo (Fig. 1A). On CEUS performed one month after MWA, there was no echo inside the fibroids and an inhomogeneous echo was present around the lesion, which was caused by contrast perfusion. This indicated the presence of a residue inside the fibroids, which required re-ablation (Fig. 1B). Uterine fibroids displayed a rapid wash-in and slow wash-out enhancement, as seen from the TIC of CEUS (Fig. 1C). The reason for this was that the RT of uterine fibroids was shorter than that of normal uterine muscle.

In a representative subject of the control group, conventional US prior to MWA revealed a circular lump with clear margins and homogeneous echo in the uterus. CDFI displayed striped or ring-shaped blood signals inside the fibroids (Fig. 1D). On conventional US performed half a year after MWA, fibroids appeared with an inhomogeneous echo inside and no blood signal, indicating that the lesion had been completely ablated (Fig. 1E).

From these results, it may be deduced that CEUS provides information on the blood supply and perfusion of fibroids prior to MWA. This may be used to evaluate the effectiveness of MWA immediately after the therapy. Re-ablation is required if remnants of fibroids are verified.

On MRI, the non-perfused area of the fibroids prior to and after MWA was distinctly displayed (Fig. 2). In a representative subject from the experimental group, uterine fibroids demonstrated high signal intensity with distinct boundaries and regular shapes on contrast-enhanced T1WI one week prior to MWA (Fig. 2A). One month after the MWA therapy, contrast-enhanced T1WI displayed fibroids with hypointensity inside and periphery enhancement (Fig. 2B).

In a representative subject from the control group, multiple uterine fibroids measuring 45x40x43 mm with mixed high signal intensity were detected on sagittal T2WI one week prior to MWA (Fig. 2C). Fibroids appeared as hypointense with clear boundaries and no remnant was seen on T2WI half a year after MWA. The reduction of fibroids in volume was estimated to be $80 \%$ (Fig. 2D).

The signal intensity on MRI was closely associated with the proton component of the lesion. Therefore, MRI cannot 
Table IV. Evaluation of the effect of microwave ablation on uterine fibroids by CEUS and MRI.

\begin{tabular}{|c|c|c|c|c|c|c|}
\hline \multirow[b]{2}{*}{$\begin{array}{l}\text { Imaging } \\
\text { method }\end{array}$} & \multicolumn{3}{|c|}{ Experimental group (n) } & \multicolumn{3}{|c|}{ Control group (n) } \\
\hline & $\begin{array}{l}\text { Total } \\
\text { fibroids }\end{array}$ & $\begin{array}{l}\text { Complete fibroid } \\
\text { regression }\end{array}$ & Residualfibroid & $\begin{array}{l}\text { Total } \\
\text { fibroids }\end{array}$ & $\begin{array}{l}\text { Complete } \\
\text { fibroid regression }\end{array}$ & Residualfibroid \\
\hline CEUS & 78 & 71 & 7 & 83 & 71 & 12 \\
\hline MRI & 78 & 67 & 11 & 83 & 64 & 19 \\
\hline P-value & - & 0.01 & $<0.01$ & - & 0.03 & 0.01 \\
\hline$\chi^{2}$ & & 8.14 & & & 3.27 & \\
\hline
\end{tabular}

CEUS, contrast-enhanced ultrasound; MRI, magnetic resonance imaging.
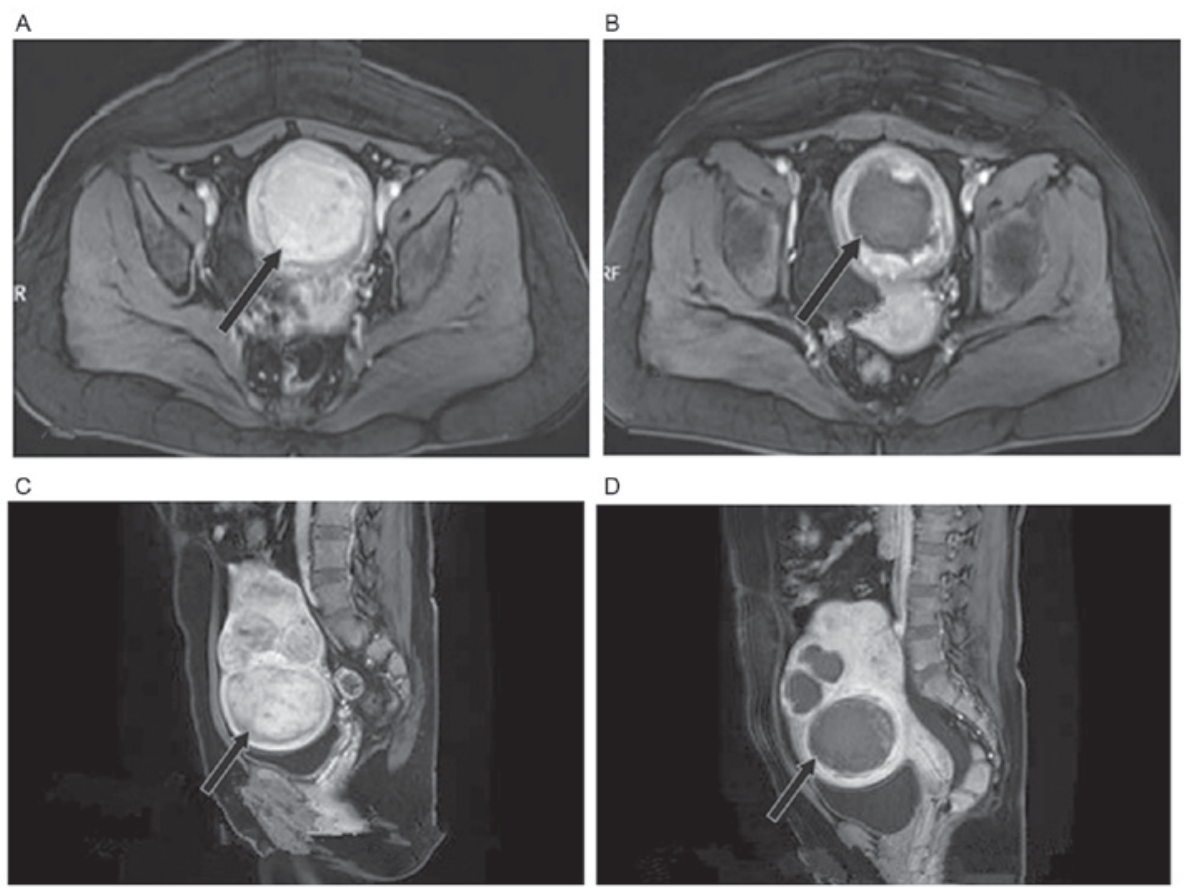

Figure 2. Magnetic resonance images of (A and B) a patient from the experimental and (C and D) a patient from the control groups. (A) T1WI at one week prior to MWA. (B) Partial enhancement was confirmed in the margin of the fibroid on T1WI acquired one month after MWA (arrow). (C) Multiple fibroids appeared with mixed hyperintensity on T2WI with the largest one measuring 45x40x43 mm (arrow). (D) Fibroids were found to be hypointense with distinct boundaries on T2WI (arrow). There was no residue and the reduction in volume was estimated to be $80 \%$. T1WI, T1-weighted imaging; MWA, microwave ablation.

present real-time blood supply of the lesion and it is not optimal for immediate evaluation after MWA.

Effectiveness of CEUS in the follow-up of patients after $M W A$. A total of 78 single or multiple fibroids were confirmed by pre-operative CEUS. Three months after MWA, CEUS revealed no perfusion inside or around the lesion in 71 fibroids. Contrast-enhanced MRI displayed no enhancement in the targeted area in 67 fibroids, indicating a complete inactivation. However, abnormal contrast perfusion was found in 7 fibroids, while mild enhancement was verified in 11 fibroids, indicating incomplete ablation and partial remission.

At the end of the follow-up period, significant differences were observed between residual ratios on MRI and CEUS in each group and complete fibroid regression was observed (Table IV). However, no significant difference was noted between the experimental and control groups in the regression and residual ratios, suggesting that MVA is effective in treating uterine fibroids. It was noted that CEUS is better than MRI in evaluating regression and residual ratios.

\section{Discussion}

Conventional two-dimensional US and CDFI are commonly used to assess the efficacy of MWA treatment of uterine fibroids through displaying the size, internal echo changes and blood supply of fibroids (18). However, the limitations lie in determining the area of fibroid necrosis as well as fibroid regression and residue. CEUS and contrast-enhanced MRI reveal the perfusion changes inside the treated area and blood supply of the fibroids, evaluate the fibroid regression and effectiveness objectively and guide targeted touch-up procedures in a timely manner to obtain better results (19). 
In the present study, CEUS and MRI were performed for a comparative investigation on the perfusion in the targeted region and the ablation area after MWA (20). According to the results obtained by examining parameters during different phases on CEUS, a significant difference was observed in the STD between vessel and tissue in fibroids, WiR of fibroid tissue and RT and WiR ratios of vessel to tissue in fibroids. The intensity of fibroid tissue had a steeply increasing curve, arrived at the PE rapidly and then slowly decreased on TIC. It displayed a fast wash-in and slow wash-out enhancement on CEUS due to the anatomical link with the surrounding normal muscle fibers and perfusion of fibroids. The enhancement inside the fibroids was greatly reduced after MWA compared with that prior to MWA. The higher the signal intensity on T1WI and T2WI, the lower the reduction in volume. A significant difference was noted in the volume reduction of fibroids with different blood supply and signal intensity.

Based on the results of the present study, signal intensity changes in the targeted region one month after MWA demonstrated coagulation necrosis in fibroids. The CEUS findings, including the maximum diameter and volume of fibroids and non-perfused region, were consistent with the MRI findings regarding the necrotic area. However, CEUS is advantageous over MRI, as it effectively displays the maximum diameter and volume of fibroids following MWA. Therefore, CEUS is an effective and reliable imaging technique in evaluating short-term results of MWA.

The incidence of pain in the sacrococcygeal and treated regions, and skin burns encountered in the present study conformed with those reported by previous studies (21). These adverse events are common after MWA, and should be paid attention to and avoided. No complications were observed in the present study, indicating that CEUS it is safe for real-time evaluation of the treatment of uterine fibroids by MWA.

The present study revealed a significant difference in fibroid regression and residual ratios between CEUS and MRI. Treatment results were associated with the fibroid size, ablation time and the surgeon. Large fibroids usually present with abundant internal blood supply and fast blood flow. Heat loss during MWA leads to fibroid residue. Real-time evaluation during MWA therapy is required to re-ablate fibroid residue in order to obtain better results.

In conclusion, CEUS is advantageous over MRI, as it safely provides real-time information on the perfusion. It reliably provides information on the ablation area and fibroid residue after MWA. Therefore, it is of great value in clinical practice and may be utilized for assessing the efficacy of MWA treatment of uterine fibroids.

\section{Acknowledgements}

This study was supported by The Regional Special Disease Center Construction Project of Zhejiang Province (no. 2014-98).

\section{References}

1. Talaulikar VS and Manyonda I: Progesterone and progesterone receptor modulators in the management of symptomatic uterine fibroids. Eur J Obstet Gynecol Reprod Biol 165: 135-140, 2012.
2. Olejek A, Olszak-Wąsik K and Czerwinska-Bednarska A: Long-term intermittent pharmacological therapy of uterine fibroids-a possibility to avoid hysterectomy and its negative consequences. Prz Menopauzalny 15: 48-51, 2016.

3. Carrafiello G, Recaldini C, Fontana F, Ghezzi F, Cuffari S, Laganà $\mathrm{D}$ and Fugazzola $\mathrm{C}$ : Ultrasound guided radiofrequency thermal ablation of uterine fibroids: Medium-term follow-up. Cardiovasc Intervent Radiol 33: 113-119, 2010.

4. Ankem K: Information-seeking behaviour of women in their path to an innovative alternate treatment for symptomatic uterine fibroids. J Med Libr Assoc 95: 164-172, 2007.

5. Estrade-Huchon S, Bouhanna P, Limot O, Fauconnier A and Bader G: Severe life-threatening hemoperitoneum from posttraumatic avulsion of a pedunculated uterine leiomyoma. J Minim Invasive Gynecol 17: 651-652, 2010.

6. Ellens $\mathrm{N}$ and Hynynen K: Simulation study of the effects of near- and far-field heating during focused ultrasound uterine fibroid ablation using an electronically focused phased array: A theoretical analysis of patient safety. Med Phys 41: 072902, 2014.

7. Chen XM and Luo PF: Current status, questions and challenges of transcatheter uterine artery embolization for the treatment of uterine fibroids. J Int Radio 15: 449-450, 2006.

8. Ren XL, Zhou XD, Zhang J, He GB, Han ZH, Zheng MJ, Li L, $\mathrm{Yu} \mathrm{M}$ and Wang L: Extracorporeal ablation of uterine fibroids with high-intensity focused ultrasound: Imaging and histopathologic evaluation. J Ultrasound Med 26: 201-212, 2007.

9. Ghezzi F, Cromi A, Bergamini V, Scarperi S, Bolis P and Franchi M: Midterm outcome of radiofrequency thermal ablation for symptomatic uterine myomas. Surg Endosc 21: 2081-2085, 2007.

10. Dong BW, Liang P, Yu XL, Yu DJ, Zhang J, Feng L, Cheng ZG, Wang Y and Wang ZL: Long-term results of percutaneous sonographically-guided microwave ablation therapy of early-stage hepatocellular carcinoma. Zhonghua Yi Xue Za Zhi 86: 797-800, 2006 (In Chinese).

11. Liang P, Wang Y, Zhang D, Yu X, Gao Y and Ni X: Ultrasound guided percutaneous microwave ablation for small renal cancer: Initial experience. J Urol 180: 844-848, 2008.

12. Zhang J, Feng L, Zhang B, Ren J, Li Z, Hu D and Jiang X: Ultrasound-guided percutaneous microwave ablation for symptomatic uterine fibroid treatment-a clinical study. Int $\mathrm{J}$ Hyperthermia 27: 510-516, 2011.

13. Zhang J, Feng L, Zhang BS, Ren JT, Li ZC, Wen B, Hu DM, Jiang $X$ and Du LD: The study of percutaneous microwave coagulation for uterine myomas. Chin J Med Ultrasound (Electronic Edition) 8: 40-43, 2011.

14. Wang F, Zhang J, Han ZY, Cheng ZG, Zhou HY, Feng L and Hu DM: Imaging manifestation of conventional and contrast-enhanced ultrasonography in percutaneous microwave ablation for the treatment of uterine fibroids. Eur J Radiol 81: 2947-2952, 2012.

15. Huang JL, Xiong WL, Deng LW and Cheng GX: The diagnostic value of MRI in adenomyosis. Chin J CT \& MRI 23: 1417-1419, 2015.

16. Wei CF, Hu B and Jiang LX: Evaluation of uterine adenomyoma treatment with high intensity focused ultrasound by contrast-enhanced ultrasound. Chin J Med Ultrasound (Electronic Edition) 7: 54-59, 2010.

17. Zhu Li, Chen WZ, Chen JY, Wen HY, Deng YB, Zhang R and Wang ZB: Study on the relationship between ultrasound ablation for uterine fibroids and signal of MRI. Acta Acad Med Militaris Tertiae 14: 1370-1373, 2009.

18. Hindley J, Gedroyc WM, Regan L, Stewart E, Tempany C, Hynyen K, Mcdannold N, Inbar Y, Itzchak Y, Rabinovici J, et al: MRI guidance of focused ultrasound therapy of uterine fibroids: Early results. AJR Am J Roentgenol 183: 1713-1719, 2004.

19. Zhou XD, Ren XL, Zhang J, He GB, Zheng MJ, Tian X, Li L, Zhu T, Zhang M, Wang L and Luo W: Therapeutic response assessment of high intensity focused ultrasound therapy for uterine fibroid: Utility of contrast-enhanced ultrasonography. Eur J Radiol 62: 289-294, 2007.

20. Zhang X, Peng S, Chen JY and Zhang RT: Investigation on influence factors of ultrasound ablation in treating multiple uterine fibroids. J Minimally Invasive Med 03: 2014.

21. Yan LM, He J, Huang GH, He M, Li KQ and Zhang L: High intensity focused ultrasound ablation for uterine fibroids in patients with retroposition of uterus. Zhongguo Chao Sheng Yi Xue Zazhi 28: 72-74, 2012. 